\title{
Le routage IP statique avec Marionnet : retour d'expérience
}

\author{
Rushed Kanawati, Jean-Vincent Loddo \\ prénom.nom@iutv.univ-paris13.fr \\ Département Réseaux \& Télécommunication, IUT de Villetaneuse \\ Université Paris 13 \\ 99 av. J.B. Clément, 93430 Villetaneuse, France
}

\begin{abstract}
RESUME : Dans cet article nous rapportons notre expérience concrète d'utilisation du logiciel Marionnet pour l'enseignement du routage IP statique. La maîtrise du routage statique nécessite habituellement de réaliser des montages relativement complexes qui mobilisent de nombreuses ressources (ordinateurs, routeurs). Ces ressources sont forcément à partager entre les étudiants, ce qui crée des situations qui limitent l'efficacité de l'enseignement. Notre expérience avec la virtualisation des réseaux montre, en revanche, l'intérêt d'une telle approche pour l'enseignement de sujets complexes et exigeants en terme de ressources.
\end{abstract}

Mots clés : réseaux, routage statique, virtualisation, Marionnet.

\section{INTRODUCTION}

La maîtrise du routage statique dans le réseau Internet est un des principaux objectifs pédagogiques de l'enseignement des réseaux informatiques aux étudiants des IUT en semestre 2 en département Réseau \& Télécommunication (R\&T). Les objectifs de cet enseignement sont principalement :

1. apprendre aux étudiants l'élaboration de règles de routage et l'édition des tables de routage des machines ;

2. apprendre la configuration des équipements d'interconnexion de réseaux (i.e. notamment les routeurs) ;

3. comprendre le déroulement des protocoles annexes nécessaires au bon fonctionnement de l'échange des paquets IP [5], en particulier le protocole de signalisation ICMP [6] et le protocole de résolution d'adresses ARP [7]. Le but principal ici est d'apprendre aux étudiants l'interprétation des messages ICMP afin de localiser et corriger des erreurs/pannes de configuration et/ou d'interconnexion des réseaux.

Le programme pédagogique national [3] des départements R\&T en France accorde ${ }^{1}$ une place importante aux séances de travaux pratiques dans l'enseignement des réseaux informatiques. À titre d'exemple, le module $R 4$ : technologie IP a un volume total de 60 heures par étudiant, dont 30 heures sont des travaux pratiques. Pour remplir complètement les objectifs pédagogiques cités ci-dessus, il est nécessaire, dans l'idéal, de réaliser des montages physiques relativement complexes. Ensuite, sur cette base, il est nécessaire de provoquer des scénarii instructifs d'un point de vue pédagogique : les paquets IP devront traverser plusieurs réseaux et subir éventuellement des erreurs de routage. Il est évident que la mise en place de telles maquettes

1 à juste titre selon l'expérience des auteurs de cet article pose de nombreux problèmes de montage à cause essentiellement du nombre important de ressources physiques (i.e. ordinateurs et équipements d'interconnexion) déployées par étudiant. La solution que nous présentons dans cet article a été expérimentée avec succès au département R\&T de l'IUT de Villetaneuse, depuis maintenant deux ans, dans différentes formations (niveau DUT 1ère et 2ème année, niveau Licence Professionnelle). Elle consiste à utiliser l'application Marionnet [1] permettant, sur une simple machine équipée d'un système GNU/Linux, de « virtualiser » des salles de travaux pratiques de réseaux. Avec Marionnet il est en effet possible d'avoir une infrastructure de travail individuelle, où chaque étudiant dispose, devant son PC, de tout l'arsenal nécessaire à la réalisation d'un certain exercice : des machines (virtuelles), des répéteurs (hub), des commutateurs (switchs), des routeurs, des câbles droits ou croisés. Sur son réseau virtuel, l'étudiant est seul maître à bord et devra assembler et configurer la totalité des composants constituant le réseau.

Dans cet article nous rapportons notre expérience d'utilisation de Marionnet pour un sujet spécifique, celui du routage statique. Dans un souci pédagogique, nous montrons d'abords comment l'enseignement de ce sujet était mis en place dans une salle classique de travaux pratiques. Nous soulignerons les limites principales du dispositif classique, puis nous montrerons comment le sujet est abordé aujourd'hui à l'aide de Marionnet et quelles sont les améliorations concrètes constatées.

\section{TP DE ROUTAGE CLASSIQUE}

Dans cette section nous décrivons l'enseignement du routage statique tel qu'il était dispensé dans nos formations avant l'introduction du logiciel Marionnet. Dans un premier temps, nous décrivons l'infrastructure, assez typique, de la salle réseaux employée (section 2.1). Ensuite nous décrivons le sujet de travaux pratique qui était proposait en tenant compte des contraintes de l'infrastructure disponible. Le principaux incon- 
vénients du sujet de TP seront ensuite commentés dans la section 2.3 .

\subsection{Description des équipements}

Le département R\&T de l'IUT de Villetaneuse bénéficie d'une infrastructure assez typique pour la réalisation de séances de travaux pratique en réseaux. Les séances sont organisées en groupes réduits, jusqu'à 18 étudiants, et se déroulent dans des salles spécialisées, équipées d'environ 20 ordinateurs de type PC. Une première différence avec une salle informatique ordinaire est que chaque ordinateur est équipé de deux cartes réseaux Ethernet. Les ordinateurs sont disposés en rangées et chaque machine dispose, à proximité, de deux prises Ethernet déportées : la première est reliée à une baie de brassage contenant autant de concentrateurs (hub) que de rangées, et la deuxième est relié à un commutateur (switch) branché au réseau général de l'IUT. Nous disposons également d'un nombre réduits d'équipements d'interconnexion de réseaux : une dizaine de commutateurs et quelques routeurs (environ 5). Un système d'installation et de diffusion d'image disque est installé. Ainsi, chaque enseignant a la possibilité d'installer, sur l'ensemble des machines de la salle, le système d'exploitation souhaité en fonction du sujet à traiter. Pour l'ensemble de nos sujets de TP réseaux nous employons un système GNU/Linux distribué par Debian [8].

\subsection{Un TP de routage}

Le sujet du routage statique est généralement planifié après une série de séances de travaux pratiques introductifs où les étudiants apprennent à manipuler les commandes de base d'administration d'interfaces réseaux, la manipulation simple de table de routage, l'interconnexion de deux réseaux Ethernet et la configuration un routeur logiciel de type Quagga [4]. Le sujet proposé consiste à réaliser deux réseaux par rangée LAN_1i et LAN_2i où i est le numéro de la rangée. D'une part, les deux réseaux LAN_1i et LAN_2i sont interconnectés par un routeur et, d'autre part, tous les réseaux LAN2i sont interconnectés via un réseau transversal qui relie ainsi toutes les rangées. Les étudiants sont répartis en groupes et chaque groupe s'occupe de la configuration des deux réseaux de leur rangée. La figure suivante illustre la maquette finale du TP (les carrés représentent des ordinateurs et les cercles des routeurs).

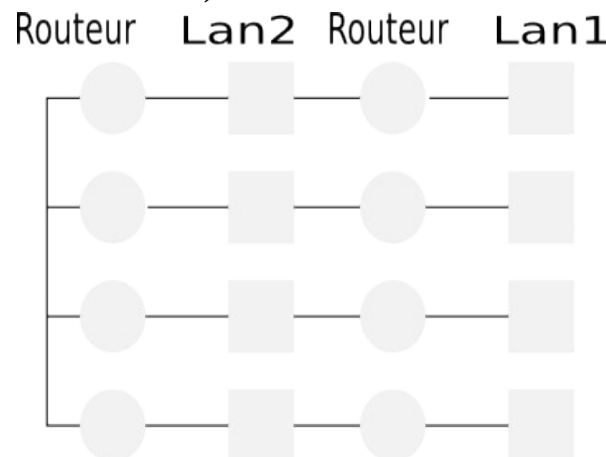

Fig. 1. Montage du TP classique sur le routage statique
Dans ce montage, le chemin le plus complexe entre deux machine nécessite de traverser au plus quatre routeurs.

\subsection{Critique du sujet}

Le sujet décrit a été expérimenté et amendé pendant plusieurs années. Bien que sa complexité reste assez mesurée, le déroulement des séances TP n'étais pas entièrement satisfaisant. En effet, le sujet présente deux inconvénients majeurs difficilement évitables (en raison des contraintes de l'infrastructure et des ressources disponibles) :

1. au sein d'un groupe d'étudiants (composé de 3 à 4 étudiants) le niveau de difficultés des différentes sous-tâches est très hétérogènes. Par exemple, la configuration du deuxième routeur (i.e routeur 2) dans chaque rangé est sans doutes la tâche la plus technique ; cette tâche incombe souvent à l'étudiant plus habile, pendant que les autres s'occupent des tâches plus simples ;

2. l'avancement d'un groupe dépend de l'avancement des autres groupes ;

3. la configuration des routeurs reliés au réseau transversal nécessite une bonne coordination entre les différents groupes (notamment le câblage et l'attribution d'adresses) ; malgré des consignes bien claires de la part des enseignants, cette tâche reste source de beaucoup d'erreurs et pertes de temps ; il s'agit d'un exercice de coordination certainement formateur pour le travail en groupe, mais cela n'est pas l'objectif de ce TP.

L'introduction du logiciel Marionnet nous a permis non seulement de faire disparaître ces inconvénients mais, aussi, nous a permis de proposer un sujet de TP plus riche et plus instructif pour les étudiants. Nous décrivons ce sujet dans la section suivante.

\section{LE ROUTAGE AVEC MARIONNET}

\subsection{Marionnet : description générale}

Marionnet est un "laboratoire de réseaux virtuels » $[1,2]$ livré sous licence GPL v2 et disponible pour téléchargement depuis le site web du projet http://www.marionnet.org. Marionnet est une sur-couche d'un principe de virtualisation appelé User Mode Linux (UML) [10]. Il permet d'exécuter sur un seul ordinateur (PC ou MAC Intel) équipé d'un système GNU/Linux, un réseau virtuel d'ordinateurs, d'appareils divers (répéteurs ou hubs, commutateurs ou switch, routeurs) et de câbles RJ45. La simulation étant extrêmement fidèle, le travail qu'un étudiant effectue sur le réseau virtuel est généralement identique à celui qui aurait lieu dans des vrais conditions, ce qui offre plusieurs avantages d'ordre pédagogique, notamment dans des modes d'enseignement à distance (la définition et l'état d'un réseau virtuel entier pouvant être empaquetés dans un mail de quelques méga-octets). 


\subsubsection{Montage d'un réseau virtuel}

Marionnet permet de réaliser des montages de réseaux, de lancer, puis de contrôler les composants en exécution à travers une interface graphique (cf. Fig. 2), conçue essentiellement pour un public d'étudiants. Depuis son introduction dans l'enseignement réseau de notre IUT, on constate que les étudiants sont capables de prendre en main le logiciel très rapidement, en quelques minutes, après les explications de base sur son fonctionnement.

Pour commencer un montage on crée un nouveau projet. Dès lors, des icônes correspondant aux équipements utilisables deviennent disponibles sur une palette à gauche de la fenêtre principale. En cliquant sur une icône de cette palette on peut ajouter un équipement à notre salle virtuelle, ou éditer les caractéristiques d'un équipement déjà introduit. On peut aussi lancer suspendre, réveiller ou arrêter tout type de composant de notre salle virtuelle. Au centre de la fenêtre principale est dessiné automatiquement un schéma réseau représentant l'état courant de salle. Un onglet étiqueté interfaces permet de pré-configurer à travers le logiciel, si on le souhaite, les interfaces réseaux des machines et des routeurs avant leur lancement.

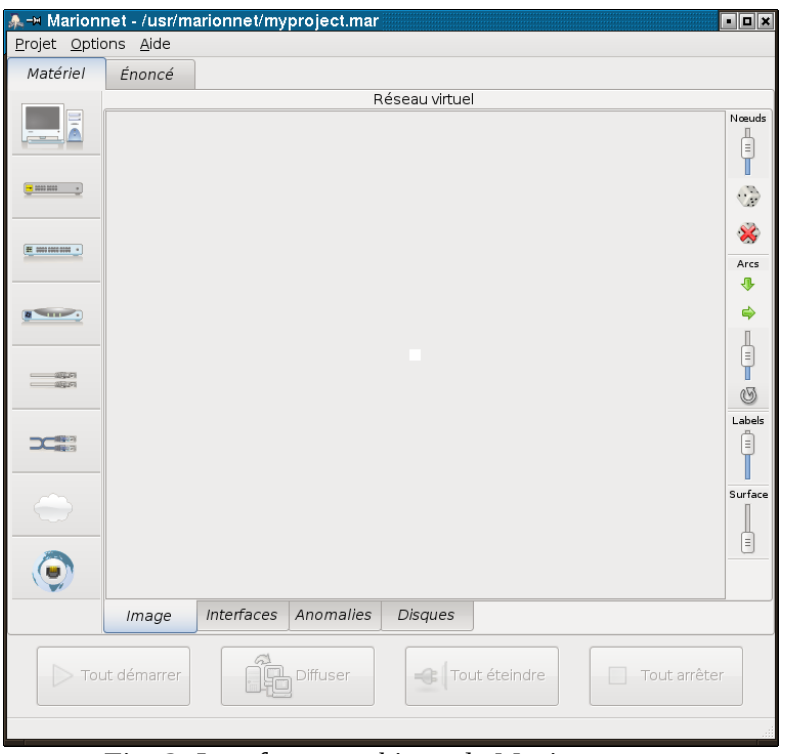

Fig. 2. Interface graphique de Marionnet

\subsubsection{L'interaction avec les composants}

Une fois le montage terminé, on peut lancer l'ensemble des composants en cliquant sur le bouton " tout démarrer ». Des fenêtres représentant les différents équipements seront alors affichés. Pour les machines virtuelles, la fenêtre contiendra un terminal sur lequel sera lancé un interpréteur de commandes (un shell bash). Pour les matériels d'inter-connexion (hub, switch, routeur), une petite fenêtre graphique avec des leds (un pour chaque port) permettra d'observer en temps réel le trafic sur les ports du matériel en question, tout comme on peut faire avec un vrai équipement.

Dans la version actuelle de Marionet, le routeur n'a pas une interface qui lui est propre. Son administra- tion se fait par une connexion Telnet (sur le port 2601) à partir d'une machine connectée au routeur dans le réseau virtuel. Les routeurs sont en réalité des machines virtuelles «déguisées » en routeur sur lesquelles tourne une instance (ou démon) du logiciel Quagga [4]. Ce démon écoute sur les ports conventionnels d'administration des routeurs et, une fois la connexion telnet établie, répond au langage de commandes IOS (langage défini et utilisé par la société CISCO et devenu standard de fait).

\subsection{Le routage avec Marionnet}

Le TP de routage avec Marionnet est aussi l'occasion de présenter pour la première fois le logiciel aux étudiants.

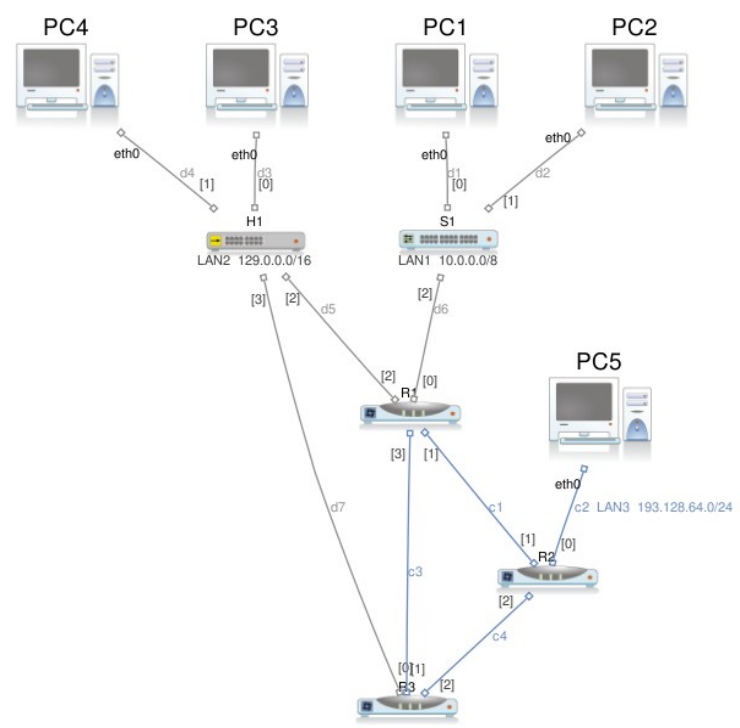

Fig. 3. Montage de base avec Marionnet

En effet, nous avons choisi de faire travailler les étudiants sur ce sujet après la série de TP introductifs classiques (voir section 2.2). L'idée est de leur permettre de travailler avec des équipements physiques (notamment pour le câblage) avant de commencer à manipuler une environnement virtuel où tout est extrêmement plus simple et rapide. L'expérience des années précédentes nous a encouragé à ne pas réserver une séance complète pour la prise en main du logiciel, l'interface graphique du logiciel s'étant révélée très intuitive et simple d'utilisation. Un tel sujet, nécessitant un montage plus complexe que les montages réalisés dans les séances précédentes, permet à lui seul de justifier l'introduction de Marionnet. Les étudiants se retrouvent devant un moyen de contourner le problème de la disponibilité limitée du matériel physique et acceptent donc de jouer le jeu de bon gré. La figure 3 . illustre le montage qu'on demande aux étudiants de réaliser avec Marionnet.

Nous reproduisons ci-après un large extrait du sujet proposé. Pour certaines questions du TP nous soulignons ici l'objectif pédagogique. Ce texte, non explicité dans le texte d'origine, sera par la suite formaté en italique et entre parenthèses. Le montage comprend trois réseaux locaux interconnectés par trois routeurs : 
1. LAN1 est un réseau local commuté comprenant les deux stations PC1 et PC2. À ce réseau est attribuée la plage IP 10.0.0.0/8. Le réseau est servi par le routeur $\mathrm{R} 1$.

2. LAN2 est un réseau local partagé comprenant les deux stations PC3 et PC4. À ce réseau est attribuée la plage 129.0.0.0/16. Le réseau est servi par les routeurs R1 et R3.

3. LAN3 représente une machine isolée PC5 connectée au routeur R2. À ce réseau est attribuée l'adresse 193.128.64.0/24.

Les trois routeurs sont connectés entre eux. Une fois le montage terminé on demande d'effectuer les tâches suivantes :

1. Attribuer des adresses IP valides aux différentes interfaces et configurer les routeurs de sorte que : (1) la connectivité entre chaque paire de machines soit assurée, (2) les paquets à destination de PC3 passent par R3 et ceux à destination de PC4 passent par $\mathrm{R} 1$, (3) la règle par défaut au niveau du routeur $R_{i}$ soit d'envoyer le paquet au routeur $R_{i+1}$. (Cet exercice vise notamment à permettre aux étudiants de se familiariser avec l'interface graphique du logiciel : choix des matériels, construction des connexions et utilisation de l'onglet interface. La connexion circulaire entre les trois routeurs permet de réaliser une boucle pour les paquet à destination inconnue. Ceci permet de tester la génération des messages d'erreur signalant l'expiration de durée de vie d'un paquet IP.)

2. Sur PC5 exécuter la commande ping PC2 -c 1. Quel est le trafic observé par PC1 suite à l'exécution de cette commande ? Justifier la réponse. (L'objet de cette question de rappeler le fonctionnement d'un commutateur. Noter qu'un commutateur dans l'environnement Marionnet est implémenté en utilisant le composant logiciel vde-2 [9].).

3. Quel est l'adresse MAC source de la trame reçue par PC2 qui encapsule le paquet Echo-request envoyé par PC5 ? Justifier la réponse.

4. Sur PC5 exécuter la commande ping PC3 -c 1. Quel est le trafic observé par PC4 suite à l'exécution de cette commande?

5. Quel est l'adresse MAC source de la trame reçue par PC3 et qui encapsule le paquet Echo-request envoyé par PC5 ?

6. Sur PC5 exécuter la commande ping 4๑.28.๑.1 - c 1 . Justifier le trafic observé.

Chaque étudiant doit travailler le sujet individuellement pour une durée de 4h30. Ainsi, chaque étudiant est confronté à tous les problèmes et doit réaliser toutes les tâches conduisant à leur solution.

\subsubsection{Bilan}

Le sujet a été soumis à quatre groupes d'environ 15-18 étudiants pour un échantillon qui dépasse donc les 60 étudiants. La quasi totalité des étudiants a réussi à trai- ter la totalité du sujet dans le temps imparti. En effet, malgré le nombre relativement élevé de composants réseau (21 composants, câbles inclus), l'exécution du logiciel se déroule d'une manière très satisfaisante, sans temps d'attente observables. Nous avons ainsi vérifié que cette solution permet bien de s'affranchir des limites imposées par la disponibilité des ressources physiques, et de répondre donc à l'ensemble des limitations évoquées dans la section 2.3.

\subsubsection{Reproductibilité du TP}

Un projet Marionnet (fichier .mar) est téléchargeable à l'adresse :

http: //www.marionnet.org/download/exercises/pl an-et-enonce-cetsis-2010.mar

Cette archive, que le logiciel permet d'ouvrir, contient à la fois le plan du réseau virtuel (machines, appareils, câblage) et l'énoncé complet du TP au format PDF (accessible par l'onglet Documents). Pour l'installation du logiciel, suivre les indications à l'adresse :

http://wiki.marionnet.org/doku .php?id=documentation:installing_marionnet

\section{CONCLUSION}

Dans cet article nous avons discuté l'intérêt pédagogique du logiciel Marionnet pour l'enseignement pratique du routage statique. La maîtrise du concept de routage nécessite de travailler sur des montages complexes qui nécessitent la disponibilité d'un grand nombre de ressources physiques. On réagit naturellement en partageant les ressources disponibles entre les étudiants, ce qui limite l'efficacité de l'enseignement. La virtualisation, c'est-à-dire la capacité de simuler sur un seul ordinateur l'ensemble des ressources constituant un réseau, est une solution à ce problème. Par ailleurs, la facilité d'utilisation de Marionnet et les bonnes performances du logiciel (et des sous-couches UML et vde sur lequel il repose) est un facteur qui renforce la pertinence de cette solution. Nous remarquerons enfin que les étudiants peuvent s'exercer en dehors des séances habituelles de travaux pratiques, ce qui ne pouvait pas être possible auparavant.

\section{Bibliographie}

[1] Jean-Vincent Loddo, Marionnet: un logiciel graphique pour l'apprentissage et l'enseignement des réseaux locaux d'ordinateurs: Premier Workshop pédagogique "Réseaux \& Télécoms”, Saint-Pierre de la Réunion, 2007.

[2] Jean-Vincent Loddo, Luca Siau. Marionnet: a virtual network laboratory and simulation tool: SimulationWorks, Marseille (France), 2008.

[3] Programme Pédagogique National du DUT Réseaux et Télécommunication.http://www.iutrt.net/IMG/pdf_PPN_Resea ux_Telecommunication_Rentree08_32400.pdf

[4] http://www.quagga.net

[5] http://www.ietf.org/rfc/rfc791.txt

[6] http://www.ietf.org/rfc/rfc792.txt

[7] http://www.ietf.org/rfc/rfc826.txt

[8] http://www.debian.org

[9] http://www.virtualsquare.org

[10] http://user-mode-linux.sourceforge.net 